\title{
The effect of forced choice on color discrimination'
}

MICHAEL H. SIEGEL, ALBION COLLEGE, Albion, Michigan 49224

Color discrimination ability was measured at four spectral regions usually named blue, green, yellow, and red. Two methods, temporal forced-choice and phenomenal report, were compared. Data suggest that for color discrimination, phenomenal report is a preferable technique.

Selecting the best instrument for measurement is a problem for all science. Psychophysics, though, has been continually concerned with finding methods to measure various sensitivities with as little contamination as possible. That method which eliminates all subjective bias and allows the measurement of pure sensory capacity is still the holy grail to crusading psychophysicists.

Hue discrimination can be investigated by a variety of methods. In an earlier study (Siegel, 1962), the methods of adjustments, limits, and constant stimuli were compared following suggestions made by Blackwell (1952) for assessment of different psychophysical methods. Blackwell proposed that the adequacy of a procedure was defined by reliability, inferred validity, and sensory determinacy. The last term referred to the absolute size of the estimate of sensitivity. The better the sensitivity, the more sensory determinate the measure. It was found, both from a statistical and a logical analysis, that of the three psychophysical methods, the method of constant stimuli was most appropriate for measuring color discrimination.

Examination of Blackwell's work on the forced-choice procedure (1952) suggested this as another and possibly better method for hue discrimination work. The contention was that the usual methods used for gathering data for brightness discrimination admitted bias. When Os were required to respond to a stimulus with a phenomenal report of "yes" or "no" there was more opportunity for the introduction of bias than with a forced-choice procedure. Blackwell further reported that a forced choice involving temporal intervals rather than spatial locations was superior. It certainly is the case that the O's task is not complicated when he is required to say "yes" or "no" under the usual conditions of a constant stimulus method. Nothing about this situation should be expected a priori to introduce bias systematically. If it were established, however, that a forced-choice procedure was most appropriate for hue discrimination, it should be used for future work.

There are many differences be tween Blackwell's study and the hue-discrimination problem. In addition to his concern with detection of light instead of difference in hue, Blackwell used a procedure in which luminances were ordered into blocks of 20 or 40 of the same magnitude. It was, in part, because of the lack of randomization that the use of yes-no categories led to a bias that Blackwell terms channelization. It is unclear whether or not the same findings would apply to a hue-discrimination study in which stimulus order was randomized.

The point of the present investigation is to evaluate the forced-choice technique in the context of a hue-discrimination problem.

\section{METHOD}

\section{Apparatus}

Two Bausch and Lomb 500-mm monochromators presented the stimulus, which appeared as a vertically divided circular field of light, $3 \mathrm{deg}$ in diam at $O$ 's eye. The area surrounding the stimulus field was white and was illuminated from above and behind the $O$ by a source whose luminance was $1 \mathrm{ft}-\mathrm{L}$ and whose color temperature matched llluminant $\mathrm{C}$. The $\mathrm{O}$ sat with his head in a head and chin rest and looked at the stimulus in Maxwellian view through an artificial pupil. Stimulus exposure, controlled by a sector shutter, was set to $.2 \mathrm{sec}$ throughout the study. The luminance of each standard stimulus, which appeared on the left half of the divided field, was set to $1 \mathrm{ft}$-L. Calibrations performed both before and after experimentation on wavelength, luminance, and stimulus duration showed no changes in preexperimental values.

\section{Observers}

There were five Os. Two were highly trained in psychophysical observation; three had no previous experience. All were color normal as determined by the Farnsworth-Munsell test. Four Os were emmotropic and one was myopic. The myopic's data are included since they do not differ systematically from the other Os'. Each viewed the stimulus with the right eye; the left eye was occluded.

\section{Procedure}

Hue discrimination was studied at four spectral locations: 480 , 520,580 , and 620 millimicrons. Most Os, when restricted to a single color name, label these blue, green, yellow, and red, respectively.

For both the phenomenal report and the forced-choice situations, a constant stimulus method with five different variable stimulus steps was employed. Before each experimental session, a pretest was run. This was done to determine the wavelength settings of the five variable stimuli and to permit the $O$ to match the brightness of each of these stimuli to that of the standard. A session consisted of 50 judgments in which the variable stimulus was longer in wavelength than the standard and another 50 judgments in which the variable stimulus was shorter.

For the phenomenal report sessions, Os were shown the range of variable stimuli and asked to say "yes" when the stimulus differed from the standard in the appropriate color direction and "no" when it did not. In the forced-choice situation, four .2-sec stimuli were successively flashed. In three of these, the variable stimulus was set as close to the standard as possible. Only in the fourth was the variable stimulus set to one of the five prearranged wavelengths. The O's task was to report which of the four flashes contained this variable stimulus.

The results are derived from four separate sessions conducted at each wavelength for both procedures. Data were first collected from two Os; some months later, the study was replicated with the three remaining Os. Since there were no significant differences in the data from the two sessions, the results were combined.

\section{RESULTS AND DISCUSSION}

Results for forced-choice and phenomenal report are treated identically except that the forced-choice data were first corrected by the equation

$$
P=\left(P^{\prime}-C\right) /(1-C)
$$

where $\mathbf{P}$ is the corrected proportion, $\mathbf{P}^{\prime}$ is the raw proportion, and $\mathrm{C}$ is the proportion of chance success, $25 \%$ in this case. Unlike Blackwell's study, where the limen was used to estimate sensitivity, here the standard deviation of the normalized 
Table 1

Comparison of Standard Deviations of Forced Choice and Phenomenal Report Methods

\begin{tabular}{llrrrr}
\hline & & \multicolumn{4}{c}{ Wavelength in Millimicrons } \\
\cline { 3 - 6 } Observer & & 480 & 520 & 580 & 620 \\
\hline AW & Phen. Rep. & 1.07 & 1.00 & .55 & .93 \\
& For. Ch. & 1.09 & 1.83 & .82 & 1.55 \\
MC & P.R. & .74 & 1.18 & .80 & 1.09 \\
& F.C. & .83 & 2.39 & .89 & 1.80 \\
EB & P.R. & .88 & 1.67 & 1.13 & 2.60 \\
& F.C. & 1.00 & 2.28 & 1.05 & 2.43 \\
WM & P.R. & 1.57 & 2.40 & .75 & 1.29 \\
& F.C. & 1.79 & 3.30 & 1.24 & 1.50 \\
LC & P.R. & .93 & 1.78 & .72 & 1.68 \\
& F.C. & 1.32 & 2.65 & 1.53 & 2.16 \\
Mean for & P.R. & 1.04 & 1.61 & .79 & 1.52 \\
all Os & F.C. & 1.21 & 2.49 & 1.11 & 1.89 \\
\hline
\end{tabular}

response distribution was used. Siegel (1962) discusses the use of the standard deviation as an alternative to the limen.

Table 1 presents the data for all Os for both procedures. In all cases but two, the phenomenal report results in lower standard deviation values and therefore better sensitivity than the forced-choice method. Both forced-choice and phenomenalreport data were highly reliable and no preference for methods may be found by comparing inferred validity. If we may judge the adequacy of the technique by Blackwell's third criterion (sensory determinacy), phenomenal report is preferable to forced choice for color discrimination.

Parallel movement of the data across wavelengths suggests that the problem for the $\mathrm{O}$ was similar in the two situations. The data suggest that the forced-choice situation is more difficult than the phenomenal report. This impression is reinforced by the comments of all Os. The fact that each forced-choice session was about four times as long as the phenomenal-report sessions probably had an influence.

On the basis of this study, we may conclude that for hue discrimination, unlike the case for brightness discrimination, phenomenal report is preferable to forced choice.

\section{REFERENCES}

BLACKWELL, H. R. Studies of psychophysical methods for measuring visual thresholds. Joumal of the Optical Society of America, 1952, 42, 606-616.

SIEGEL, M. H. Discrimination of color. I. Comparison of three psychophysical methods. Journal of the Optical Society of America, $1962,52,1067-1070$.

NOTE

1. Results of part of this experiment were reported at the 1968 meeting of the Eastern Psychological Association. The research was supported in part by Public Health Service Grant MH-14281. 